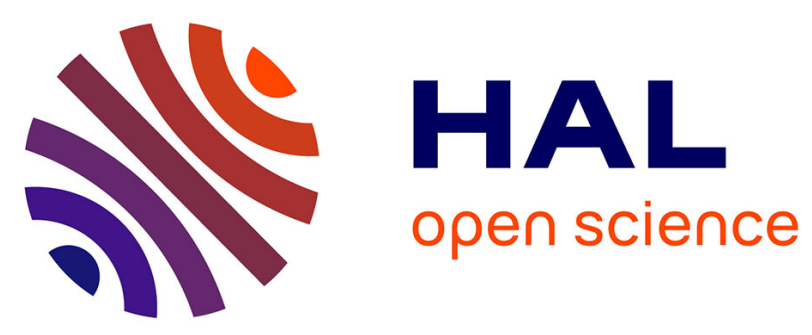

\title{
Learning a CNN on multiple sclerosis lesion segmentation with self-supervision
}

Alexandre Fenneteau, Pascal Bourdon, David Helbert, Christine Fernandez-Maloigne, Christophe N Habas, Rémy Guillevin

\section{To cite this version:}

Alexandre Fenneteau, Pascal Bourdon, David Helbert, Christine Fernandez-Maloigne, Christophe N Habas, et al.. Learning a CNN on multiple sclerosis lesion segmentation with self-supervision. 3D Measurement and Data Processing, IS\&T Electronic Imaging 2020 Symposium, Jan 2020, San Francisco, United States. 10.2352/ISSN.2470-1173.2020.17.3DMP-002 . hal-02378210

\section{HAL Id: hal-02378210 https://hal.science/hal-02378210}

Submitted on 7 Feb 2020

HAL is a multi-disciplinary open access archive for the deposit and dissemination of scientific research documents, whether they are published or not. The documents may come from teaching and research institutions in France or abroad, or from public or private research centers.
L'archive ouverte pluridisciplinaire HAL, est destinée au dépôt et à la diffusion de documents scientifiques de niveau recherche, publiés ou non, émanant des établissements d'enseignement et de recherche français ou étrangers, des laboratoires publics ou privés. 


\title{
Learning a CNN on multiple sclerosis lesion segmentation with self-supervision.
}

\author{
Alexandre Fenneteau ${ }^{1,2,3}$, Pascal Bourdon ${ }^{2,3}$, David Helbert ${ }^{2,3}$, Christine Fernandez-Maloigne ${ }^{2,3}$, Christophe Habas ${ }^{1,3}$, Remy \\ Guillevin $^{3,4,5}$ \\ 1) Neuroimaging Department, Quinze Vingts Hospital; Paris, France \\ 2) XLIM Laboratory, University of Poitiers, UMR CNRS 7252; Poitiers, France \\ 3) I3M, Common Laboratory CNRS-Siemens, University and Hospital of Poitiers ; Poitiers, France \\ 4) Poitiers University Hospital, $\mathrm{CHU}$; Poitiers, France \\ 5) DACTIM-MIS/LMA Laboratory University of Poitiers, UMR CNRS 7348; Poitiers, France
}

\begin{abstract}
Multiple Sclerosis (MS) is a chronic, often disabling, autoimmune disease affecting the central nervous system and characterized by demyelination and neuropathic alterations. Magnetic Resonance $(M R)$ images plays a pivotal role in the diagnosis and the screening of MS. MR images identify and localize demyelinating lesions (or plaques) and possible associated atrophic lesions whose MR aspect is in relation with the evolution of the disease.

We propose a novel MS lesions segmentation method for MR images, based on Convolutional Neural Networks (CNNs) and partial self-supervision and studied the pros and cons of using self-supervision for the current segmentation task. Investigating the transferability by freezing the firsts convolutional layers, we discovered that improvements are obtained when the CNN is retrained from the first layers. We believe such results suggest that MRI segmentation is a singular task needing high level analysis from the very first stages of the vision process, as opposed to vision tasks aimed at day-to-day life such as face recognition or traffic sign classification. The evaluation of segmentation quality has been performed on full image size binary maps assembled from predictions on image patches from an unseen database.
\end{abstract}

\section{Introduction}

Multiple sclerosis (MS) is a central nervous system autoimmune disease. It affects 1 to more than 200 in 100000 people depending on the region [1], it generally appears near 30 years old [2] and can rapidly induce high disability [3]. Magnetic resonance (MR) imaging is one of the most valuable exam for the diagnosis, prognosis and following-up of MS [4]. MR images enable to identify, localize, count and to determine activity of demyelinating lesions; this procedure appears to be a repetitive and time consuming task, and is often accomplished with computer vision-based virtual assistance with possible inter-observer variability [5].

The interest in automatic white matter (WM) lesion and especially MS lesion segmentation has grown significantly in the past decade. Several challenges such as the Medical Imaging Computing \& Computer Assisted Intervention (MICCAI) MS lesion segmentation 2016 [6] have been conducted for better performance evaluation within the computer vision community. Annotated patient datasets have been made publicly available too, making it easier to explore the capacity of machine learning algorithms such as CNNs to synthesize semantics from medical im- ages. Last researches in this field study the importance of some parameters and suggest different techniques to improve segmentation, most of them use CNNs. Nair et al. proposed in [7] to resort the Montecarlo dropout in CNN to access to segmentation indicators such as prediction variability. Roy et al. presented in [8] a convolutional network and showed improvements by augmenting patch size. Hashemi et al. adapted a 3D U-net in [9] with fully connected ones in the encoder and decoder pathes with better results and studied the influence of loss function parameters and the patch fusion strategy. Valverde et al., in [10], were interested in the reusability of their $\mathrm{CNN}$ for images from other centers with other MR scanners and protocols and showed that good results can be obtained with few new annotations and parameters fine-tuning. McKinley et al. [11] demonstrated that simultaneous segmenting WM lesions and brain tissues improves the quality of segmentation and Brosch et al. [12] pre-trained their CNN with convolutional restricted Boltzmann machines in an unsupervised way to improve segmentation performances.

All of aforementioned studies used CNN on either 2D slices or $3 \mathrm{D}$ volumes, some of them using patch and others complete slices or volumes from multi-modal MR images. However, most of them utilized a neural network architecture designed as an encoder-decoder with skip connections more or less close to a Unet [13]. Only Valverde et al. [10] considered the segmentation task as a classificication voxel by voxel.

While deep convolutional networks have gradually become a reference in computer vision, as a strongly data-driven supervised technique $[14,15]$, its use in medical imaging is often limited by the small amount of samples available. An other difficulty is to obtain expert annotations from radiologists as it requires a lot of time not always available [16]. Fortunately techniques such as data augmentation or transfer learning have been proposed to overcome such limitations [17].

Because of the great amount of unnanotated MR images available in a hospital, it was proposed to easily improve segmentation results by leveraging available unnanotated data without more annotations. Such process can be adapted for almost all techniques and tasks in MR and Computerized Tomography (CT) imaging using self-supervision.

The self-supervised technique introduced by Doersch et al. in [18] aimed at reducing the need for large numbers of annotated samples. This technique is based on context learning [19] and transfer learning [20]. It relies on training a neural network on an 
unsupervised, seemingly useless task (i.e. which does not need annotation) to learn context, and to partially reuse the resulting network to learn inference on the initial, supervised target task. As the network is already trained to understand incoming image data on a similar but simpler task, fewer annotated samples are needed.

To the best of our knowledge, only few studies as [21, 22] reported utilization of self-supervision in medical imaging analysis. Therefore, we propose a novel strategy to learn context for selfsupervision in medical imaging and to evaluate its performance for multiple sclerosis lesion segmentation.

We assume that our implementation of self-supervision can guide the supervised learning task within a CNN already designed for MR image analysis, thus reducing potentially bad solutions and improving the overall quality of segmentation.

Investigating the potential of self-supervision in MR imaging is important because this technology evolves very quickly, leading to an exponential increase of already high-dimension data spaces for which only few samples are available. In our case we expect to work on multiple MRI sequences in the coming years to study MS, starting with very few patients. In this context we will need new strategies for data augmentation or advanced model learning.

\section{Material and method}

The neural network described by Isensee et al. [23] was chosen because of its proven efficiency in segmentation. It achieved the third rank on the MICCAI Brain Tumor Segmentation (BraTS) challenge 2017 and it uses an encoder-decoder architecture with skip connections as in most of recent papers in medical imaging segmentation. We adapted it to take five 3D patches as input, respectively extracted from the T1 weighted (T1W), T1 weighted with contrast enhancing agent (T1Wc), T2 weighted (T2W), T2weighted-fluid-attenuated inversion recovery (FLAIR), and proton density weighted (PDW) images as such sequences are generally analyzed by the radiologist to evaluate the presence of multiple sclerosis lesions.

The initial, unsupervised target task aimed at data selflearning is dedicated to predicting the location of a local patch over the full image. We expect this multi-output regression task to share self-trained features with a model dedicated to brain lesion segmentation. Our architecture for segmentation is an encoderdecoder, so we only trained the encoder part, as no reconstruction is needed for this task. The encoder had to predict the $\mathrm{x}, \mathrm{y}$ and $\mathrm{z}$ coordinates of one patch lying in the whole image. The trained encoder weights were transferred to the segmentation encoder for the supervised task of MS lesion segmentation. During training, the segmentation was performed and evaluated on patches. The method is summarized on Figure 1.

We worked with normalized images aligned to each other and resized to $128 \times 128 \times 128$ voxels. Patch size is set to $32 \times 32 \times 32$, which is a good trade-off between having lesions with their surrounding environment and having sub-images small enough to emulate sample data augmentation. Such a choice has already shown good results with Roy et al. [8]. Our CNN were not trained with data augmentation since it drastically increases the time of training even if it has been shown to improve results. Samples missing a given input modality (e.g. T1Wc or PDW) in the dataset were given a zero-valued image for substitution, and the sequence dropout technique detailed in [24] was also applied.
This technique consists in randomly setting input modalities to zero in order to ensure the $\mathrm{CNN}$ fitting model is generic enough to provide reliable predictions even when modalities are missing. We apply repeated random sub-sampling cross-validation method during training. A batch consists of 12 randomly selected patches of the same patient with at least $75 \%$ of brain avoiding to take into account patches without brain. The $\mathrm{CNN}$ is trained to maximize the Dice score (equation 3), which is a compromise between precision (equation 2 and sensibility 1 as detailed in equation 4 aimed at reducing the impact of unbalanced data distribution on result evaluation. The Adam [25] optimizer is used helping reducing the time of convergence.

$$
\begin{aligned}
& \text { Sensitivity }=\frac{T P}{P} \\
& \text { Precision }=\frac{T P}{T P+F P} \\
& \text { Dicescore }=\frac{2 T P}{2 T P+F P+F N} \\
& \text { Dicescore }=2 \cdot \frac{\text { Precision } \cdot \text { Sensitivity }}{\text { Precision }+ \text { Sensitivity }}
\end{aligned}
$$

\section{Results}

Four different public data sets of multiple sclerosis lesion segmentation were gathered, namely the MICCAI MS lesion segmentation 2008 [26] and 2016 [6], the International Symposium on Biomedical Imaging (ISBI) 2015 MS segmentation challenge [5] consisting of 21 exams from 5 different patients at different time points, and the public data set of Lesjak et al. [27]. Two other public data sets from a manually selected subset of OASIS3 [28] with cognitively normal and declining patients and MICCAI BraTS challenge 2017 [29] with brain tumors were used as additional image source. More informations about datasets are shown in the table 1. MICCAI 2016 dataset was kept as the test dataset ensuring results to be acquired with as MR input images as possible.

Table 1 : Details of the datasets used

\begin{tabular}{|l||l|l|l|}
\hline Dataset & Exams & MR images & MS \\
\hline MICCAI 2008 & 20 & T1W, T2W and FLAIR & YES \\
\hline MICCAI 2016 & 15 & $\begin{array}{l}\text { T1W, T1Wc, T2W, FLAIR } \\
\text { and PDW }\end{array}$ & YES \\
\hline ISBI 2015 & 21 & $\begin{array}{l}\text { T1W, T2W, FLAIR and } \\
\text { PDW }\end{array}$ & YES \\
\hline Lesjak et al. & 30 & $\begin{array}{l}\text { T1W, T1Wc, T2W and } \\
\text { FLAIR }\end{array}$ & YES \\
\hline OASIS3 & 82 & $\begin{array}{l}\text { T1W, T2W and FLAIR } \\
\text { BraTS 2017 }\end{array}$ & $\begin{array}{l}\text { T1W, T1Wc, T2W and } \\
\text { FLAIR }\end{array}$ \\
\hline
\end{tabular}

All images from datasets were preprocessed to be as comparable as possible. The preprocessing is really close to the one performed by [5] for the ISBI challenge. When it was possible, the unprocessed images were used. After the first N4 bias field correction [30], we chose to register all images to the FLAIR image as most of all lesions from our datasets were segmented into 
1)
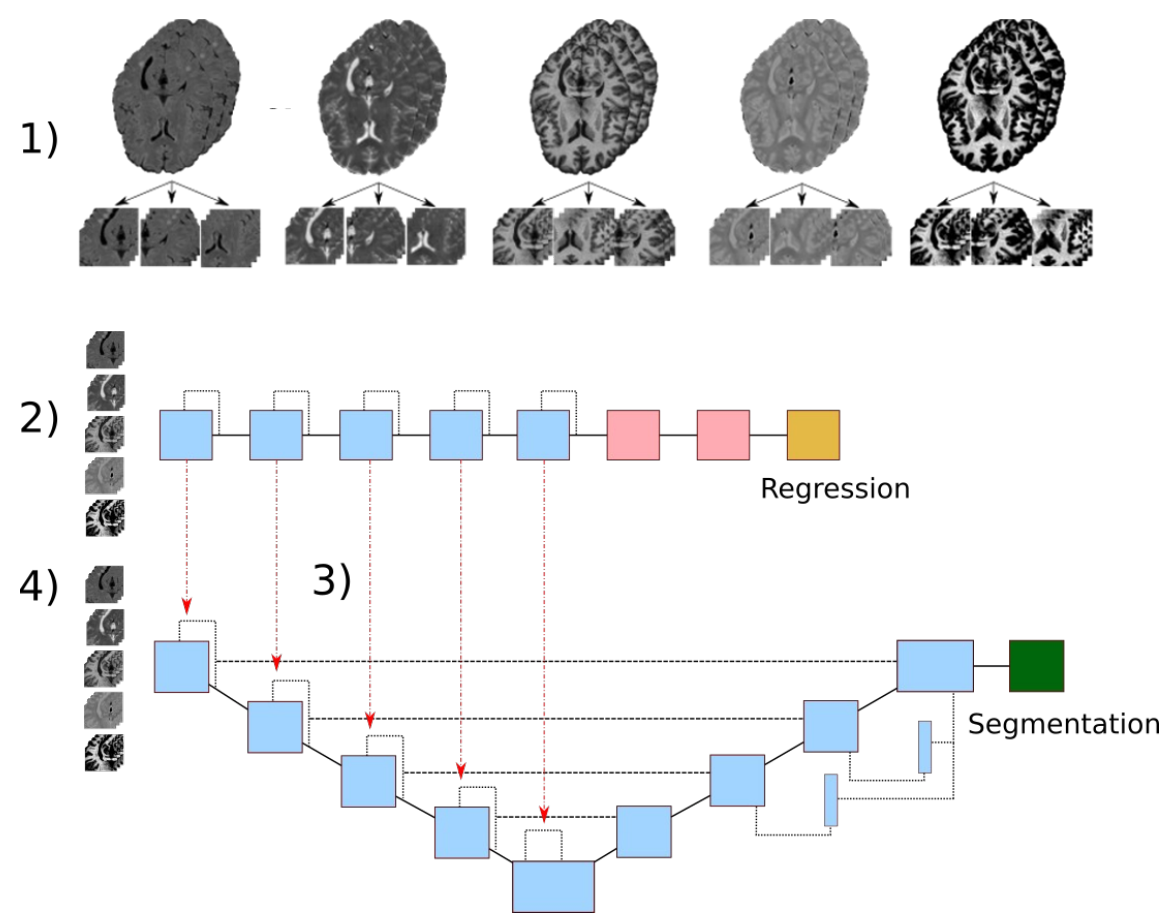

Figure 1. MS lesion segmentation workflow: 1) Patches are extracted from whole images. 2) Patches are sent to the first neural network for training on the localization task 3) Layer weights are transferred to the segmentation CNN. 4) Training on the segmentation task.

the FLAIR space. The registration were performed by the FSL FLIRT [31, 32, 33] tool. Before skull stripping [34], the histogram matching [35] were applied for each modality separately. The references images for histograms were chosen among good looking images from our datasets at this step of preprocessing. The T1 image is then skull stripped and registered to a $1 \mathrm{~mm}$ MNI brain template [36]. The brain mask and the transformation are then applied to other images modalities. The global pipeline is illustrated in the Figure 2. We arbitrary resize all our images to $128 \mathrm{x} 128 \mathrm{x}$ 128 voxels with a $1.422 \times 1.703 \times 1.422 \mathrm{~mm}^{3}$ resolution to work with reasonable image size and resolution to distinguish lesions.

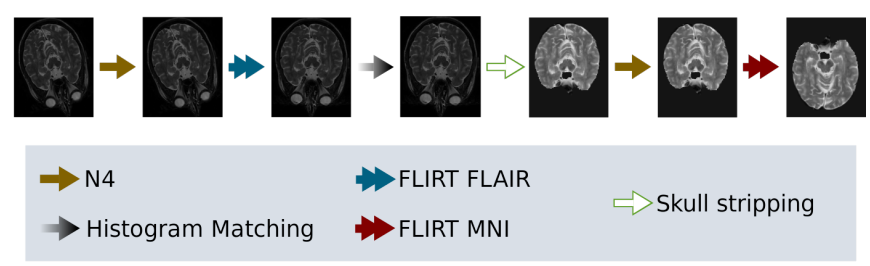

Figure 2. Step of the pipeline illustrated with a T2 image.

Pretraining on the unsupervised regression task has been performed with every training data sets, including ones containing no patients with MS. It respected a ratio of $50 \%$ of MS exams during an epoch, thus to ensure that the network can build general feature maps from different brain MRI texture avoiding the unbalance between MS and no MS exams. During the second supervised learning on lesion segmentation only MS datasets were utilized. Ground truth was randomly selected from manually annotated maps, some of which being as many as four maps for a single record depending on the number of medical experts in- volved. As manual lesion delineation tolerance can vary from one expert to the other, we assume using such individual maps instead of combinations can help the $\mathrm{CNN}$ build its own consensus.

For each patient, 150 randomly chosen patches in the brain area were segmented. Final segmentation were obtained by averaging all overlapping predictions. It ensured to have a consensus prediction covering more than two times the brain volume.

Five transfer strategies are evaluated:

- Weight transfers with the six first convolutional layers frozen $(6 \mathrm{CF})$

- Weight transfers with the three first convolutional layers frozen $(3 \mathrm{CF})$

- Weight transfers with the two first convolutional layers frozen (2CF)

- Weight transfers with the first convolutional layer frozen (1CF)

- Weights used for initialization before fine-tuning (0CF)

For comparison, we trained a vanilla version without selfsupervision only in MS lesion segmentation (VAN). The evaluation metric of similarities used for comparison is the Dice score.

The mean results are presented in table 2 and the box plot showing the distribution of results in the test set is presented in figure 3 . The evaluation was calculated on a test set acquired with different machines and protocols with ground truth made of the consensus of segmentation of different radiologists. We achieved great performance compared to recent publications keeping in mind that the test set is not the same and cannot be fully compared to other studies such as Valverde et al. [37] who obtained a Dice score of 53.5\%, Valcarcel et al. [38] with 56\% and 57\%, or Roy et 
Table 2 : Evaluation of the trainings

\begin{tabular}{|l||l|l|l|}
\hline Training & Dice score & Sensitivity & Precision \\
\hline VAN & $66.94 \%$ & $60.63 \%$ & $78.84 \%$ \\
\hline 0CF & $\mathbf{6 7 . 6 3} \%$ & $\mathbf{6 1 . 4 7 \%}$ & $79.30 \%$ \\
\hline 1 CF & $66.89 \%$ & $59.79 \%$ & $\mathbf{8 0 . 3 1 \%}$ \\
\hline 2CF & $64.44 \%$ & $57.50 \%$ & $77.53 \%$ \\
\hline 3CF & $62.69 \%$ & $55.86 \%$ & $75.06 \%$ \\
\hline 6 CF & $57.93 \%$ & $49.60 \%$ & $75.03 \%$ \\
\hline
\end{tabular}

al. [8] obtained $56.39 \%$. The good quality of segmentation for all versions indicates that the $\mathrm{CNN}$ architecture, the overall training method and the training set were adapted to our task. The best Dice score and sensitivity are reached with the $0 \mathrm{CF}$ method and the best precision is reached with $1 \mathrm{CF}$ method.
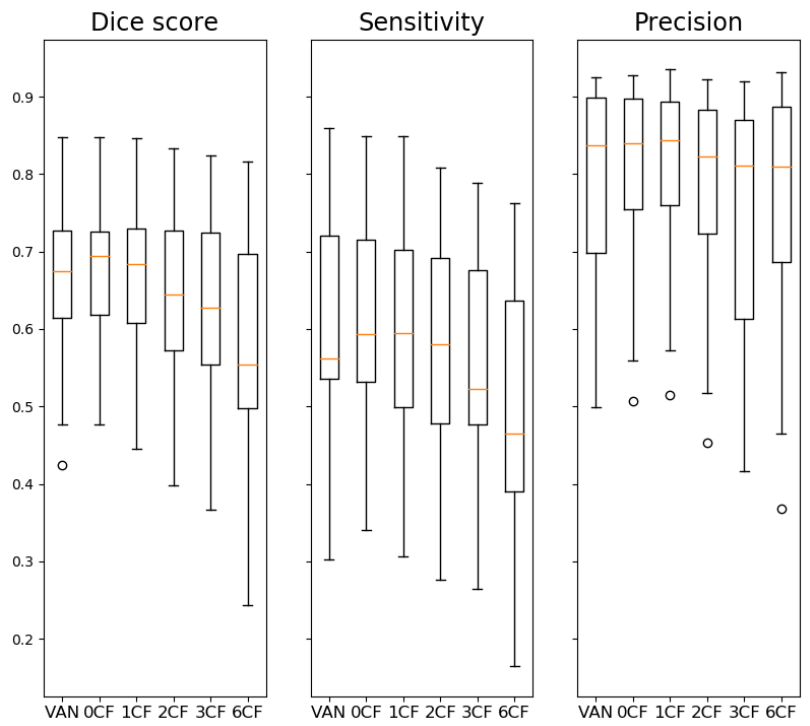

Figure 3. Box plot comparing obtained results for each learning method. Mediam is represented by the orange horizontal line.

The figure 3 shows that increasing the number of frozen layers lowers the Dice score and the sensitivity and increases the range distribution of results. The precision is also lowered with the number of frozen layers but less than the others metrics.

\section{Discussion}

Only the $0 \mathrm{CF}$ version of self-supervision outperformed for each metric the VAN method. Our initial hypothesis and motivation for self-supervision were that the unsupervised regression and supervised segmentation tasks would share the first convolutional layers of the deep network model, as expected in multiple computer vision tasks. The results however indicate that it is not the case, and that the very first layers need to be retrained for better segmentation precision. A possible hypothesis is that the MS lesion segmentation task is a very specialized one which needs a fully dedicated prediction model, as opposed to day-to-day visual object recognition or classification tasks which are assumed to share common ground.

The box plot in figure 3 shows that freezing the convolutional layers decreased the Dice score and the sensitivity from the beginning. The loss of precision seems to appear only after the freezing of the two first convolutional layers. Those results suggest that the freezing of the first convolutional layers wood mainly affect the sensitivity of the CNN. So, this is in favour with the assumption that the first layers of the CNN would capture much of the useful image information in the very first layers. It also suggests that selection and gathering of most discriminative features are achieved gradually after the first layer.

A visualisation of segmentation achieved with our technique is illustrated in figure 4 . We can see in this picture that augmenting the number of frozen layer decreased the size of segmented area and increases the size of area corresponding to false positive in the ellipse at the left frontal border of the ventricle. It can reveal underfitting because this area usually contains lesions.

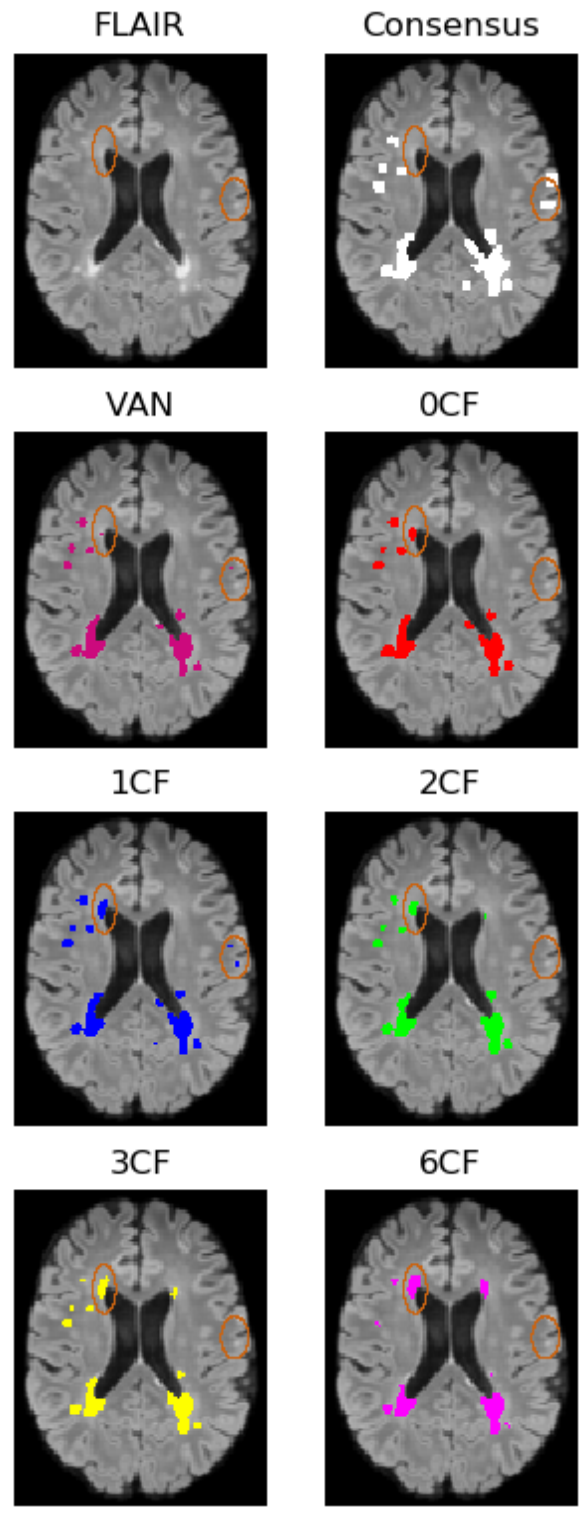

Figure 4. Example of visualisation of segmentations. Main areas of differences are delineated by orange ellipses. 
Our results gives further support to the idea that regression and segmentation task are really different tasks even if they are performed with close images. One explaining reason may be that the size of the training set was to large to observe benefits of pretraining. Observations agree with hypothesis stated in the article by Yosinski et al. [39]. It is advanced that the specialization of the neural network increases with the depth and that transfer learning seems to always provide improvements even after finetuning from the first layer. Unlike the method we propose, the two first layers are not general for both tasks but Yosinski et al. detailed two very close classification tasks and our study is on regression and segmentation task.

\section{Conclusion}

We propose a novel way to implement and use selfsupervision methods in medical imaging, using localization of partial image content as an unsupervised task, in order to reuse the trained hyperparameters to fine-tune a supervised task of interest. We obtained good overall results compared to the state of the art. Our technique improves the quality of MS lesion segmentation, not yet as much as expected, and not that way it should. We observed that even the first layers of our CNN appear to be specialized for the segmentation task and are not as general as it can be thought. First layers seems to be more implied in the sensitivity of our $\mathrm{CNN}$, indicating that the discrimination of meaningful information are conducted after those layers.

Our conclusions support the idea that using self-supervision for high-level human vision tasks such as medical imaging diagnosis is not as straightforward as it is for day-to-day vision tasks. Further investigation should be conduced to define the limits of vision task similarity and hierarchy. However, we believe that selfsupervision techniques can be more used and that finding good first unsupervised task to learn can conduce to great improvements in medical image and should be more used in this field.

\section{References}

[1] M. Pugliatti, S. Sotgiu, and G. Rosati, "The worldwide prevalence of multiple sclerosis," Clinical neurology and neurosurgery, vol. 104, no. 3, pp. 182-191, 2002.

[2] E. D. ACHESON, "EPIDEMIOLOGY OF MULTIPLE SCLEROSIS," British Medical Bulletin, vol. 33, pp. 9-14, 011977.

[3] I. Kister, E. Chamot, A. R. Salter, G. R. Cutter, T. E. Bacon, and J. Herbert, "Disability in multiple sclerosis: a reference for patients and clinicians," Neurology, vol. 80, no. 11, pp. 1018-1024, 2013.

[4] A. J. Thompson, B. L. Banwell, F. Barkhof, W. M. Carroll, T. Coetzee, G. Comi, J. Correale, F. Fazekas, M. Filippi, M. S. Freedman, et al., "Diagnosis of multiple sclerosis: 2017 revisions of the mcdonald criteria," The Lancet Neurology, vol. 17, no. 2, pp. 162-173, 2018.

[5] A. Carass, S. Roy, A. Jog, J. L. Cuzzocreo, E. Magrath, A. Gherman, J. Button, J. Nguyen, F. Prados, C. H. Sudre, et al., "Longitudinal multiple sclerosis lesion segmentation: resource and challenge," NeuroImage, vol. 148, pp. 77-102, 2017.

[6] O. Commowick, F. Cervenansky, and R. Ameli, "Msseg challenge proceedings: Multiple sclerosis lesions segmentation challenge using a data management and processing infrastructure," 2016.

[7] T. Nair, D. Precup, D. L. Arnold, and T. Arbel, "Exploring uncertainty measures in deep networks for multiple sclerosis lesion detection and segmentation," in International Conference on Medical Im- age Computing and Computer-Assisted Intervention, pp. 655-663, Springer, 2018.

[8] S. Roy, J. A. Butman, D. S. Reich, P. A. Calabresi, and D. L. Pham, "Multiple sclerosis lesion segmentation from brain mri via fully convolutional neural networks," arXiv preprint arXiv:1803.09172, 2018.

[9] S. R. Hashemi, S. S. M. Salehi, D. Erdogmus, S. P. Prabhu, S. K. Warfield, and A. Gholipour, "Asymmetric loss functions and deep densely-connected networks for highly-imbalanced medical image segmentation: Application to multiple sclerosis lesion detection," IEEE Access, vol. 7, pp. 1721-1735, 2019.

[10] S. Valverde, M. Salem, M. Cabezas, D. Pareto, J. C. Vilanova, L. Ramió-Torrentà, A. Rovira, J. Salvi, A. Oliver, and X. Lladó, "One-shot domain adaptation in multiple sclerosis lesion segmentation using convolutional neural networks," NeuroImage. Clinical, p. 101638,2018 .

[11] R. McKinley, R. Wepfer, F. Aschwanden, L. Grunder, R. Muri, C. Rummel, R. Verma, C. Weisstanner, M. Reyes, A. Salmen, et al., "Simultaneous lesion and neuroanatomy segmentation in multiple sclerosis using deep neural networks," arXiv preprint arXiv:1901.07419, 2019.

[12] T. Brosch, L. Y. W. Tang, Y. Yoo, D. K. B. Li, A. Traboulsee, and R. Tam, "Deep 3d convolutional encoder networks with shortcuts for multiscale feature integration applied to multiple sclerosis lesion segmentation," IEEE Transactions on Medical Imaging, vol. 35, pp. 1229-1239, May 2016.

[13] O. Ronneberger, P. Fischer, and T. Brox, "U-net: Convolutional networks for biomedical image segmentation," in MICCAI, vol. 9351 of LNCS, pp. 234-241, Springer, 2015. (available on arXiv:1505.04597 [cs.CV]).

[14] A. Voulodimos, N. Doulamis, A. Doulamis, and E. Protopapadakis, "Deep learning for computer vision: A brief review," Computational intelligence and neuroscience, vol. 2018, 2018.

[15] W. Rawat and Z. Wang, "Deep convolutional neural networks for image classification: A comprehensive review neural computation," 2017.

[16] G. Litjens, T. Kooi, B. E. Bejnordi, A. A. A. Setio, F. Ciompi, M. Ghafoorian, J. A. van der Laak, B. van Ginneken, and C. I. Snchez, "A survey on deep learning in medical image analysis," Medical Image Analysis, vol. 42, pp. 60 - 88, 2017.

[17] A. S. Lundervold and A. Lundervold, "An overview of deep learning in medical imaging focusing on mri," Zeitschrift für Medizinische Physik, vol. 29, no. 2, pp. 102-127, 2019.

[18] C. Doersch, A. Gupta, and A. A. Efros, "Unsupervised visual representation learning by context prediction," in Proceedings of the IEEE International Conference on Computer Vision, pp. 14221430, 2015.

[19] J. Sun and D. W. Jacobs, "Seeing what is not there: Learning context to determine where objects are missing," in Proceedings of the IEEE Conference on Computer Vision and Pattern Recognition, pp. 57165724, 2017.

[20] S. Pan and Q. Yang, "A survey on transfer learning. ieee transaction on knowledge discovery and data engineering, 22 (10)," 2010.

[21] L. Chen, P. Bentley, K. Mori, K. Misawa, M. Fujiwara, and D. Rueckert, "Self-supervised learning for medical image analysis using image context restoration," Medical image analysis, vol. 58, p. 101539, 2019.

[22] W. Bai, C. Chen, G. Tarroni, J. Duan, F. Guitton, S. E. Petersen, Y. Guo, P. M. Matthews, and D. Rueckert, "Self-supervised learning 
for cardiac mr image segmentation by anatomical position prediction," arXiv preprint arXiv:1907.02757, 2019.

[23] F. Isensee, P. Kickingereder, W. Wick, M. Bendszus, and K. H. Maier-Hein, "Brain tumor segmentation and radiomics survival prediction: Contribution to the brats 2017 challenge," in International MICCAI Brainlesion Workshop, pp. 287-297, Springer, 2017.

[24] Y. Feng, H. Pan, C. H. Meyer, and X. Feng, "A self-adaptive network for multiple sclerosis lesion segmentation from multi-contrast mri with various imaging protocols.," CoRR, vol. abs/1811.07491, 2018.

[25] D. P. Kingma and J. Ba, "Adam: A method for stochastic optimization," arXiv preprint arXiv:1412.6980, 2014.

[26] M. Styner, J. Lee, B. Chin, M. S Chin, O. Commowick, H.-H. Tran, V. Jewells, and S. Warfield, "3d segmentation in the clinic: A grand challenge ii: Ms lesion segmentation,” MIDAS Journal, 112007.

[27] Ž. Lesjak, A. Galimzianova, A. Koren, M. Lukin, F. Pernuš, B. Likar, and Ž. Špiclin, "A novel public mr image dataset of multiple sclerosis patients with lesion segmentations based on multi-rater consensus," Neuroinformatics, vol. 16, pp. 51-63, Jan 2018.

[28] D. S. Marcus, A. F. Fotenos, J. G. Csernansky, J. C. Morris, and R. L. Buckner, "Open access series of imaging studies: longitudinal mri data in nondemented and demented older adults," Journal of cognitive neuroscience, vol. 22, no. 12, pp. 2677-2684, 2010.

[29] S. Bakas, H. Akbari, A. Sotiras, M. Bilello, M. Rozycki, J. S. Kirby, J. B. Freymann, K. Farahani, and C. Davatzikos, "Advancing the cancer genome atlas glioma mri collections with expert segmentation labels and radiomic features," Scientific data, vol. 4, p. 170117, 2017.

[30] N. J. Tustison, B. B. Avants, P. A. Cook, Y. Zheng, A. Egan, P. A. Yushkevich, and J. C. Gee, "N4itk: Improved n3 bias correction," IEEE Transactions on Medical Imaging, vol. 29, pp. 1310-1320, June 2010 .

[31] M. Jenkinson and S. Smith, "A global optimisation method for robust affine registration of brain images," Medical image analysis, vol. 5, no. 2, pp. 143-156, 2001.

[32] D. N. Greve and B. Fischl, "Accurate and robust brain image alignment using boundary-based registration," Neuroimage, vol. 48, no. 1, pp. 63-72, 2009.

[33] M. Jenkinson, P. Bannister, M. Brady, and S. Smith, "Improved optimization for the robust and accurate linear registration and motion correction of brain images," Neuroimage, vol. 17, no. 2, pp. 825$841,2002$.

[34] J. E. Iglesias, C. Liu, P. M. Thompson, and Z. Tu, "Robust brain extraction across datasets and comparison with publicly available methods," IEEE Transactions on Medical Imaging, vol. 30, pp. 1617-1634, Sep. 2011.

[35] L. G. Nyúl, J. K. Udupa, and X. Zhang, "New variants of a method of mri scale standardization," IEEE transactions on medical imaging, vol. 19, no. 2, pp. 143-150, 2000.

[36] V. S. Fonov, A. C. Evans, R. C. McKinstry, C. Almli, and D. Collins, "Unbiased nonlinear average age-appropriate brain templates from birth to adulthood," NeuroImage, no. 47, p. S102, 2009.

[37] S. Valverde, M. Cabezas, E. Roura, S. González-Villà, D. Pareto, J. C. Vilanova, L. Ramió-Torrentà, À. Rovira, A. Oliver, and $\mathrm{X}$. Lladó, "Improving automated multiple sclerosis lesion segmentation with a cascaded 3d convolutional neural network approach," NeuroImage, vol. 155, pp. 159-168, 2017.

[38] A. M. Valcarcel, K. A. Linn, S. N. Vandekar, T. D. Satterthwaite, J. Muschelli, P. A. Calabresi, D. L. Pham, M. L. Martin, and R. T. Shinohara, "Mimosa: an automated method for intermodal segmen- tation analysis of multiple sclerosis brain lesions," Journal of Neuroimaging, vol. 28, no. 4, pp. 389-398, 2018.

[39] J. Yosinski, J. Clune, Y. Bengio, and H. Lipson, "How transferable are features in deep neural networks?," in Advances in neural information processing systems, pp. 3320-3328, 2014.

\section{Author Biography}

Alexandre Fenneteau has obtained his Master's degree in 2019 in computational biology focused on medical image processing. His last works with the Quinze-Vingts hospital, XLIM laboratory and I3M laboratory are focused on applying deep learning techniques to MR images for helping radiologists to leverage an increasingly amount of data. He now is a PhD student of Siemens Healthineers France working in the I3M laboratory. 


\section{JOIN US AT THE NEXT EI!}

IS\&T International Symposium on

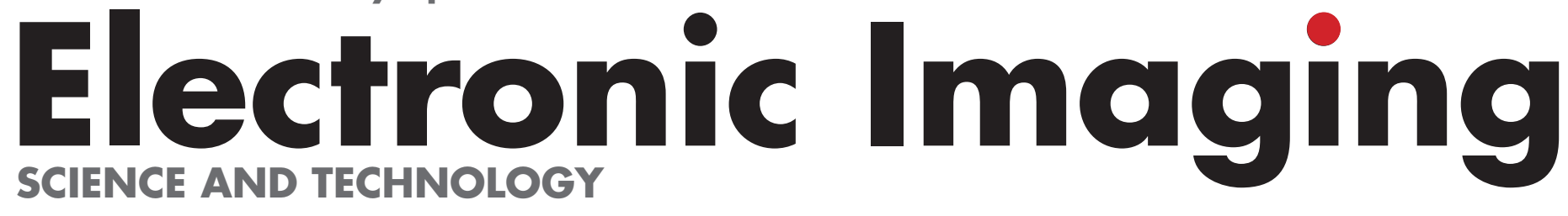

Imaging across applications ... Where industry and academia meet!
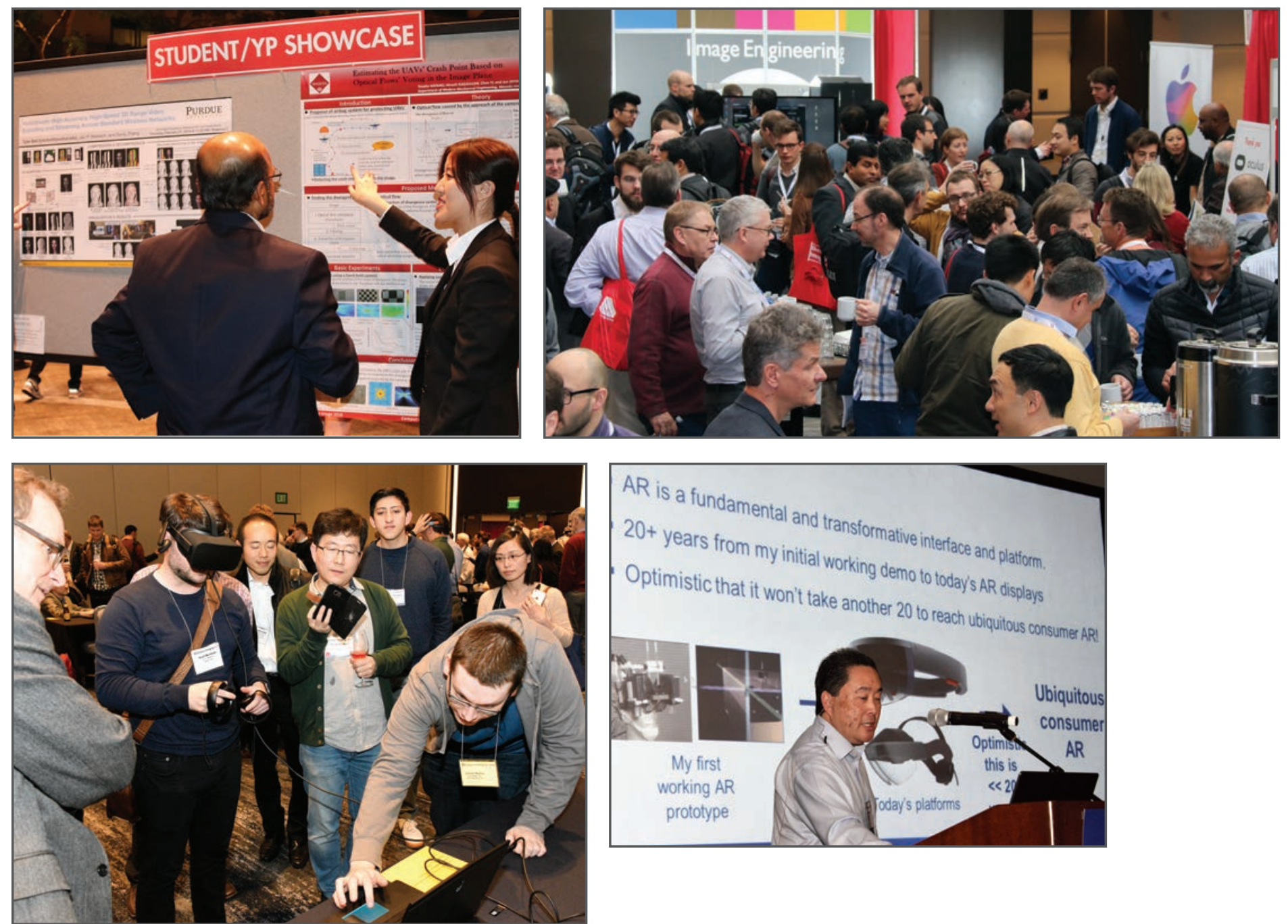

- SHORT COURSES • EXHIBITS • DEMONSTRATION SESSION • PLENARY TALKS •

- INTERACTIVE PAPER SESSION • SPECIAL EVENTS • TECHNICAL SESSIONS •

www.electronicimaging.org

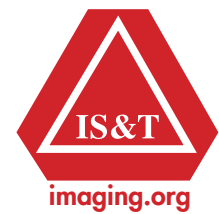

